\title{
Impact of intestinal permeability, inflammation status and parasitic infections on infant growth faltering in rural Bangladesh
}

\author{
Rie Goto*, C. G. Nicholas Mascie-Taylor and Peter G. Lunn \\ Department of Biological Anthropology, University of Cambridge, Pembroke Street, Cambridge CB2 3RA, UK
}

(Received 18 January 2008 - Revised 29 August 2008 - Accepted 1 September 2008 - First published online 24 October 2008)

A longitudinal study of 298 rural Bangladeshi infants found evidence of growth faltering starting at 3 months of age. Anthropometric status declined substantially in the first 2 years of life, with weight-for-height (WHZ) falling from -0.49 to -1.75 , weight-for-age (WAZ) from -1.18 to -2.87 and height-for-age (HAZ) from -1.00 to -1.88 . Higher concentrations of the acute-phase protein $\alpha-1$-acid glycoprotein (AGP) and higher gut mucosal damage (as signified by raised lactulose:mannitol (L:M) ratios) were both associated with chronic malnutrition as indicated by poorer HAZ and WAZ scores $(P=0.011$ and 0.005 for AGP and 0.039 and 0.019 for L:M ratio, respectively). Higher Hb levels were related to improved $z$-scores, while elevation of Giardia-specific IgM titre (GSIgM) was associated with poor WAZ and WHZ $(P=0.015$ and 0.039 , respectively). IgG did not show any significant association with $z$-scores and the L:M ratio did not correlate with any of the inflammation markers or Giardia infection. The prevalence of geohelminth infections was low (only $4 \%$ in the total study period). However, the level of GSIgM indicated high endemicity of Giardia infection from early in life, although very few cysts were detected from stool samples. These findings suggest that rural Bangladeshi infants are being exposed to high levels of infection with concomitant gut damage and growth faltering.

Infant growth: Intestinal permeability: Giardia intestinalis: Giardia-specific IgM antibody: $\alpha$-1-Acid glycoprotein

The burden of undernutrition among most young Bangladeshi children remains very high and a recent nationwide study ${ }^{(1)}$ found that $40.8 \%$ of children under 5 years old were stunted, $47.1 \%$ were underweight and $11.9 \%$ were wasted. These high burdens reflect both shorter- and longer-term insults. Growth faltering which is common in many developing countries starts at an early age with weight-for-age $z$-scores (WAZ) and weight-for-height $z$-scores (WHZ) falling from about 3 months of age until about 12 months of age, whereas the decline in height-for-age $z$-score (HAZ) starts immediately and continues until about 24 months of age ${ }^{(2)}$. The time period between 3 and 24 months of age of a child has been described as 'the window of opportunity for addressing undernutrition $^{(2,3)}$ and so interventions to improve nutritional status throughout the growth period must be accomplished within this rather short period of childhood.

A hypothesis of a cause of infant growth faltering in developing countries was suggested from work in The Gambia. Lunn $^{(4)}$ and Campbell et al. ${ }^{(5)}$ found that impaired smallintestinal mucosal function explained up to $64 \%$ of the observed height and weight faltering of Gambian infants aged between 2 and 15 months of age. They proposed that frequent gastrointestinal infections, arising from unhygienic food preparation or storage, damaged the small-intestinal mucosal function in two ways. First, by the loss of vulnerable brush-border enzymes including lactase, which impaired digestion and absorption, and second by disruption of the barrier function, which allowed translocation of macromolecules across the mucosa and into the body. Although the exact causes of the enteropathy remain unknown, the onset of mucosal damage in these Gambian infants started at about 6 months of age, which coincided with the time of introduction of homeprepared weaning foods as well as the onset of growth faltering.

Parasitic infections due to Giardia intestinalis and geohelminths are very common in many tropical environments, and these infections have been proposed as one major cause of intestinal mucosal damage ${ }^{(6-9)}$. Giardia infection is associated with poor nutritional status of young children in developing countries ${ }^{(6,10)}$ and geohelminths have also been implicated in restricting child growth ${ }^{(11)}$ and are known to cause maldigestion and malabsorption ${ }^{(12)}$. It is possible that even low intensities of infections with these parasites have marked effects on the fast-growing infant, and Trichuris trichiura has been shown to be associated with poor growth in both weight and height in Nicaraguan children less than 24 months of age ${ }^{(13)}$.

In Bangladesh, although a number of studies have examined the effect of gastrointestinal parasite infection on the growth of infants, there are little available data on infants and young

Abbreviations: AGP, $\alpha$-1-acid glycoprotein; GSIgM, Giardia-specific IgM titre; HAZ, height-for-age z-score; L:M, lactulose:mannitol ratio; WAZ, weight-for-age $z$-score; WHZ, weight-for-height $z$-score.

* Corresponding author: Dr R. Goto, fax +44 1223 335460, email rg277@cam.ac.uk 
children below 24 months of age, although a WHO study on rural Bangladeshi infants showed that $50 \%$ of 2 -year-old children had Ascaris infection, $25 \%$ Trichuris and $21 \%$ had hookworms (CGN Mascie-Taylor, personal communication). Additionally, a cross-sectional pilot survey conducted by the authors in rural Bangladesh found that $87 \%$ of 2- to 18 -month-old infants had elevated Giardia antibody titres.

The present study describes the changes in anthropometric and biochemical variables over the first 2 years of life of Bangladeshi infants and examines the inter-relationships of infant growth, Giardia infection, immune response and intestinal mucosal function.

\section{Subjects and methods}

The data came from a longitudinal randomised controlled study which examined the impact of regular anti-Giardia and anthelmintic treatments on growth, intestinal mucosal function and immune status of infants in a rural area of Bangladesh ${ }^{(14)}$. The study, however, generally failed to show any impacts of the treatments, due to the unexpectedly high rate of Giardia re-infection. The 36-week trial (June 2003 to April 2004) was carried out in Dhamrai Upazila, a typical rural area located $40 \mathrm{~km}$ north-west of Dhaka, the capital of Bangladesh. All infants under 11 months of age in the area were recruited (their age ranged from 3 to 15 months at the beginning of the study) using a list of the national immunisation programme as well as local community knowledge. After the medical doctor and the trained female local assistants explained the study purposes and the process, parental consent was obtained (a consent form was prepared in Bengali) and families were informed that they could withdraw from the study at any time.

The study was based in four contiguous administrative areas (wards) and at the beginning of the study, socio-demographic and economic information was collected from families including family size, number of siblings, religion, number of possessions (cattle, goat, television, bicycle, motorbike and mobile phone), parental background (age, occupation and education) by trained local female assistants. The Bengali questionnaire was pre-tested in the pilot study and checked for accuracy using an independent translator.

In the longitudinal study, infants had their weight and supine length measured every 4 weeks. Every 12 weeks a lactulose:mannitol (L:M ratio) intestinal permeability test was performed, a capillary blood sample was taken to measure $\mathrm{Hb}$, albumin, $\alpha$-1-acid glycoprotein (AGP), IgG and Giardiaspecific IgM titre (GSIgM) and a stool sample was collected and examined for the presence of eggs of the three common geohelminths and Giardia cysts.

\section{Anthropometry}

The infant's weight and supine length were measured using standard techniques ${ }^{(15)}$; length was measured to the nearest $0.5 \mathrm{~cm}$ using a portable anthropometric board (TALC, St Albans, Herts, UK) and weight was measured with $0.05 \mathrm{~kg}$ precision using a digital electric scale (UC-321; A \& D Co., Tokyo, Japan). Inter-observer technical error of measurement and the coefficient of reliability $(R)$ were calculated ${ }^{(16)}$ at three different times during the study period.
All values of $R$ were more than $0 \cdot 95$, so the quality of measurement was deemed acceptable throughout the study period. Most mothers remembered their infant's date of birth, as there was less than a year between the birth and their infant's recruitment into the study. Birth details were validated wherever possible using the infant's vaccination card.

\section{Urine collection for testing intestinal permeability}

The solution for testing intestinal permeability contained $200 \mathrm{mg}$ lactulose (Regulose ${ }^{\circledR}$ syrup, lactulose $3.35 \mathrm{~g}$ per $5 \mathrm{ml}$; donated by General Pharmaceuticals Limited, Gazipur, Bangladesh) and $50 \mathrm{mg}$ mannitol (Sigma-Aldrich, Poole, Dorset, UK) per $\mathrm{ml}$ water. Infants received $2 \mathrm{ml}$ of the solution per $\mathrm{kg}$ body weight using a disposable dropper with a scale so that any solution lost could be estimated and made up to ensure the infant received the correct volume. The solution is quite sweet and pleasant tasting and infants usually swallowed it readily. Mothers were requested not to feed their infants for at least $1 \mathrm{~h}$ before dosing. Infants were encouraged to drink water $30 \mathrm{~min}$ after taking the sugar solution in order to help urination. A urine specimen collection bag (Paediatric/ Non-sterile U-Bag; Hollister Limited, Aurora, ON, Canada) was attached to the infant immediately after dosing. These urine bags are equipped with a drainage tube so that urine can be removed from the bag soon after each urination, thereby keeping the infant as dry as possible and reducing loss. The urine was collected in a container marked with the infant's identification number and a few drops (about $50-100 \mu \mathrm{l})$ of $0 \cdot 2 \%(\mathrm{w} / \mathrm{v})$ chlorhexidine digluconate solution (Sigma-Aldrich) were added as a bacteriostat.

Each collection time was of $3 \mathrm{~h}$ duration, except for the first examination when urine was collected for $5 \mathrm{~h}$. If, however, the infant did not urinate within $90 \mathrm{~min}$ of taking the sugar solution, then urine collection was continued for $5 \mathrm{~h}$. The collection of urine for $5 \mathrm{~h}$ often proved to be a problem for these rural Bangladeshi mothers as they found it difficult to be absent from their house for such a long time, and $3 \mathrm{~h}$ was generally about the maximum time they could spare. Akram et al. have shown good agreement between L:M ratios collected over 2 and $6 h^{(17)}$. On completion of the urine collection and after checking for faecal contamination, total volume was measured and a $2 \mathrm{ml}$ well-mixed sample was taken and kept at $-20^{\circ} \mathrm{C}$ before shipment to the UK for analysis. If an infant had diarrhoea or skin infection of the perineal area, the test was postponed, and repeated as soon as the infant had recovered.

\section{Blood collection and $\mathrm{Hb}$ measurement}

A finger-prick technique was used to collect blood samples from the infants. This method gives minimal pain, is less invasive, causes less distress to infants, and provides sufficient blood for analysis (about $100 \mu \mathrm{l}$ ). One finger was cleaned using disinfectant liquid and, after drying completely, the finger was pricked to a depth of $2.25 \mathrm{~mm}$ using a springloaded disposable lancet (HemoCue Safety Lancet; HemoCue Ltd, Angelholm, Sweden). A maximum of five drops of blood were collected on a Guthrie card (903 Specimen Collection Paper; Schleicher \& Schuell Bioscience GmbH, Dassel, Germany). An additional drop was placed in a micro-cuvette 
(HemoCue Blood Hemoglobin Cuvette; HemoCue Ltd) and the $\mathrm{Hb}$ concentration was measured immediately using a portable haemoglobinometer (HemoCue; HemoCue Ltd). Blood spots were dried overnight at room temperature, avoiding any insect contact, and then packed individually in a sealed plastic bag and stored at $-20^{\circ} \mathrm{C}$ with desiccant before analysis.

\section{Stool sample collection and analysis}

A day before the stool sample collection, a container was distributed to the mothers, and they were instructed on how to collect a stool sample and to make sure the specimen was not contaminated with soil or dirt. Diarrhoeal stool samples were not examined as they are too diluted and another sample was taken after the infant recovered, usually within 1 to $2 \mathrm{~d}$. The collected specimens were taken as soon as possible to the laboratory in the research station where a $1.0-1.5 \mathrm{~g}$ sample was preserved in $9 \mathrm{ml}$ of $10 \%$ formalin and kept at room temperature. Stool samples were examined at the Parasitology Laboratory at the Laboratory Sciences Division of the International Centre for Diarrhoeal Disease Research, Bangladesh (Dhaka, Bangladesh) where the formalin-ether faecal concentration technique ${ }^{(18)}$ was used to measure the prevalence and intensity (eggs per g faeces) of Ascaris lumbricoides, T. trichiura and hookworm (either Ancylostoma duodenalis or Necator americanus) and cysts of $G$. intestinalis.

\section{Laboratory assays of urine and blood samples}

All urine and blood assays were conducted at the Department of Biological Anthropology, University of Cambridge (Cambridge, Cambs, UK). Enzymic assays were used to measure the concentration of mannitol, lactulose and lactose in urine ${ }^{(19,20)}$.

Plasma constituents were eluted from the Guthrie card blood-spots by $24 \mathrm{~h}$ incubation in $1.25 \mathrm{ml}$ of a phosphosaline buffer ( $0.01 \mathrm{M}$-sodium phosphate, $0.5 \mathrm{M}$-sodium chloride, $\mathrm{pH}$ $7 \cdot 2$ ) containing $1 \%$ Tween 20 . The resulting solution, a 20-to 50-fold dilution of the blood, was used directly for the analyses. An immunoturbidimetric technique was used for the albumin assay (antibody and standards supplied by DakoCytomation, Ely, Cambs, UK). Blood concentrations of IgG and AGP were determined using a standard double-sandwich ELISA (IgG antibodies from DakoCytomation and AGP antibodies from Acris Antibodies $\mathrm{GmbH}$, Hiddenhausen, Germany; standards were supplied by DakoCytomation). The Giardia-specific IgM ELISA assay used Giardia trophozoite antigen following the method of Goka et al. ${ }^{(21)}$. The $\mathrm{Hb}$ concentration in the eluted sample was analysed using the cyanmethaemoglobin method (Randox Haemoglobin Assay Kit, Randox Laboratories Limited, Crumlin, Co. Antrim, UK) and, by comparison with the $\mathrm{Hb}$ reading taken at blood collection, was used to estimate the blood volume of the eluted spot. In order to quote plasma concentrations from the blood spot measurements, a conversion factor was calculated from data in which albumin and IgG were assayed in both blood and plasma of eighty-six urban poor Bangladeshi infants. Thus, plasma concentration $=$ blood concentration $\times 3 \cdot 14$. The $\mathrm{CV}$ of all the assays were below $5 \%$. Precision of measurement was one decimal place for $\mathrm{Hb}$ and albumin and two decimal places for other biochemical variables.

\section{Statistical methods}

Data were analysed using the SPSS for Windows statistical package (versions 10 to 13; SPSS Inc., Chicago, IL, USA). Sequential multiple regression analyses were mainly used. The L:M ratio distribution was skewed and was normalised using a $\log _{10}$ transformation. Curve estimation was used to check for the linear, quadratic and cubic relationships between two continuous variables. Statistical significance was accepted at the $5 \%$ level.

HAZ, WAZ and WHZ of the infants were determined using Epi Info $^{\mathrm{TM}}$ (version 3.3.2) based on National Center of Health Statistics year 2000 references, Centers for Disease Control and Prevention ${ }^{(22)}$. Because the anthropometric measurements were not taken at exactly 4-weekly intervals, individual curve estimates (up to fourth-order polynomial) were applied and from the polynomial regressions the weights and supine lengths at $28 \mathrm{~d}$ intervals were calculated using SAS (version 8.2; SAS Institute Inc., Cary, NC, USA). The estimated weight and supine length were used to calculate the HAZ, WAZ and WHZ scores at exactly 4-weekly intervals.

GSIgM titres of infants in the present study were compared with values obtained from sixty-seven UK infants ${ }^{(23)}$ (aged 10-20 months) who were assumed not to have been exposed to Giardia. The upper $95 \%$ confidence value (mean $0.154+2 \times 0.086=0.326)$ obtained for the UK children was used as the cut-off point to delineate Giardia-positive from -negative infections in the Bangladesh study.

Ethical approval was obtained from The Bangladesh Medical Research Council.

\section{Results}

Out of the initial sample of 410 infants, 298 infants (143 males and 155 females) provided full information comprising anthropometric measurement at 4-weekly intervals (ten measurements) and blood spot, urine and stool examinations (four examinations) at 12-weekly intervals. There were no significant differences in infants' age, sex, anthropometry and socio-demographic and economic status between the sample (n 298) and those lost to attrition ( $n$ 112).

The infants' families were generally poor; most lived in houses made of mud with a tin roof $(93 \%)$, a quarter of families did not own any cattle or goats (39\%), nearly half of the fathers $(46 \%)$ worked as daily labourers and only $7 \%$ of mothers were in paid work. About half of the fathers and mothers (45 and $42 \%$, respectively) had received no formal education, and one-third of the mothers (29\%) were teenagers.

Stool examination showed that the prevalences of geohelminth infections in the infants were low (4\% in the total study period) and very few (0.9\%) of Giardia cysts were detected. However, prevalence using an elevation of GSIgM titres showed that $98 \%$ of the infants were infected with Giardia over the study period.

Table 1 presents the overall mean and standard deviation of the three $z$-scores and the six biochemical variables. The anthropometric variables provide evidence of both acute 
Table 1. Characteristics of the data of three $z$-scores and biochemical variables

(Mean values and standard deviations)

\begin{tabular}{lcc}
\hline & Mean & SD \\
\hline Age (months) & 13.2 & 4.3 \\
HAZ & -1.54 & 0.99 \\
WAZ & -2.31 & 1.24 \\
WHZ & -1.17 & 1.12 \\
Hb (g/l) & 94.9 & 13.1 \\
Albumin (g/l) & 41.7 & 6.4 \\
IgG (g/l) & 9.69 & 3.33 \\
AGP (g/l) & 0.89 & 0.36 \\
GSIgM (titres) & 2.42 & 0.92 \\
L:M ratio & & \\
Logged value & 1.18 & 0.30 \\
Geometric mean & 0.15 & \\
\hline
\end{tabular}

HAZ, height-for-age $Z$-score; WAZ, weight-for-age $z$-score; WHZ, weight-forheight $z$-score; AGP, $\alpha$-1-acid glycoprotein; GSIgM, Giardia-specific IgM titre; L:M, lactulose:mannitol.

(wasting, low WHZ) and chronic malnutrition (underweight, WAZ and stunting, HAZ) and most of the children were anaemic. IgG, which has a long half-life $(23 \mathrm{~d}$; Lydyard et al. ${ }^{(24)}$ ), reflects chronic immune stimulation. Plasma concentrations of the acute-phase protein AGP increase 3- or 4-fold within $3 \mathrm{~d}$ of infection and in the absence of further infection the half-life is about $5 \cdot 5 \mathrm{~d}^{(25)}$, so the raised AGP mean value indicates regular re-infection as do the elevated Giardia titres; high L:M ratios imply damage to intestinal mucosal function.

All $z$-scores showed a significant downward relationship with age (all $P<0 \cdot 001$; Fig. 1), with HAZ falling nearly $1 \mathrm{SD}$ from -1.00 to -1.88 , WAZ and WAZ falling more than 1.5 $\mathrm{SD}$; from -1.18 to -2.87 for WAZ and from -0.49 to -1.75 for WHZ. On average, female HAZ values were consistently higher by about one-third of an SD than males, while WHZ was higher in males $(0.22)$ on average, but the difference became smaller as the infants aged. No sex difference in WAZ was found.

$\mathrm{Hb}$ showed a slight upward relationship with age $\left(y=98.393+(-0.862) \times\right.$ age $+0.040 \times$ age $\left.^{2} ; \quad P=0.008\right)$, with values for females, on average, being $3 \cdot 1 \mathrm{~g} / 1$ higher than for males $(P<0 \cdot 001)$. Albumin showed no significant relationship with age or sex. Regression analysis showed that IgG increased by $0.26 \mathrm{~g} / 1$ per month $(P<0.001)$ and the change was consistent in both males and females. AGP showed a significant quadratic effect with age in both sexes, increasing up to about 10 months of age followed by a slight downward trend in older infants $\left(y=0.620+0.043 \times\right.$ age $+(-0.001) \times$ age $^{2}$; $P<0 \cdot 001)$. GSIgM titres showed a significant, but small, downward trend with age $(\mathrm{B}=-0.023 ; P<0.001)$. Females had higher titres on average $(\mathrm{B}=0.134 ; P=0.011)$ but the pattern was not consistent over the total age range (significant interaction effect; $P=0.004$ ) and males' GSIgM titres had a significant stronger correlation with age than females $(r 0.204$ and 0.044 , respectively, $\left.\mathrm{t}_{(\infty)}=2.799 ; P<0.01\right)$. The logged L:M ratio also showed a significant downward trend $(\mathrm{B}=-0.006$; $P=0.003)$ with, on average, females having a higher L:M ratio (B $=0.050 ; P=0.004)$.

The relationships between $z$-scores and biochemical variables were examined after controlling for age and sex effects (Table 2). Hb showed positive relationships with all $z$-scores
$(P<0.001,<0.001$ and 0.004 for HAZ, WAZ and WHZ, respectively); however, albumin showed only a positive relationship with HAZ $(P=0.016)$ but not with WAZ and WHZ. IgG failed to show any relationships with anthropometric status. The acute-phase protein AGP showed negative relationships with HAZ and WAZ $(P=0.011$ and $0 \cdot 005$, respectively) but not with WHZ. Giardia infection indicated by the elevation of GSIgM showed significant relationships with WAZ and WHZ ( $P=0 \cdot 015$ and $0 \cdot 039$, respectively). Poor intestinal mucosal function (i.e. high L:M ratio) showed significant relationships with lower HAZ and WAZ scores $(P=0.039$ and 0.019, respectively).

The inter-relationships between biochemical variables are summarised in Table 3. In general, markers of immune response of inflammation (i.e. IgG, AGP and GSIgM) tended to show significant strong positive relationships. Higher concentrations of albumin were also associated with higher concentrations of the inflammation markers. The L:M ratio, however, did not show significant association with Giardia infection or any of the immune responses.

\section{Discussion}

Childhood malnutrition remains an almost invariable characteristic of many developing countries. The causes are associated with the complex interplay between poverty, inadequate food quantity and quality, frequent infections, poor sanitary conditions, inappropriate hygiene practices and poor accessibility and affordability of health services. In addition, the capacity to digest and absorb food may be impaired by frequent and chronic illness such as protozoa and geohelminth infection in the intestine. The present study focused on the critical first 2 years of life and specifically investigated the relationship between the infants' growth status and the level of small-intestinal mucosal damage, parasite burden and the local and systemic inflammation and the immune responses.

The present study found a sharp decline in all three $z$-scores from about 3 months of age. These worsening $z$-scores over the first year of life are in agreement with three other Bangladeshi studies, two conducted in urban slums by Arifeen et al. ${ }^{(26)}$ and Karim \& Mascie-Taylor ${ }^{(27)}$ and one in a rural area by Begum $^{(28)}$, which showed improvements up to age 4-5 months followed by marked declines, particularly for HAZ and WAZ, until 12 months of age. This downward trend and the timing of failing growth status in Bangladesh are also following, in general, the findings of declines in $z$-scores in developing countries in Africa, Latin America and Asia by Shrimpton et al. ${ }^{(2)}$.

IgG levels generally increase with age in childhood ${ }^{(29)}$, especially in developing countries, and the rise is even faster as production of $\mathrm{IgG}$ in the body is stimulated by infections. Fig. 2 compares the relationship between $\operatorname{IgG}$ and age in a UK reference ${ }^{(30)}$, a Gambia study (Campbell et al. ${ }^{(5)}$; PG Lunn, personal communication) and the present study. The UK reference and the present study show remarkably similar parallel regression lines $(B=0.244$ and 0.230 , respectively) but different intercept values, with a higher level of about 3 units in the present study. The relationship with age in The Gambia was significantly steeper $(B=0.581)$ than the UK or the present study $(P=0.001$ and 0.007 , respectively), indicating that the Gambian infants were exposed to more 

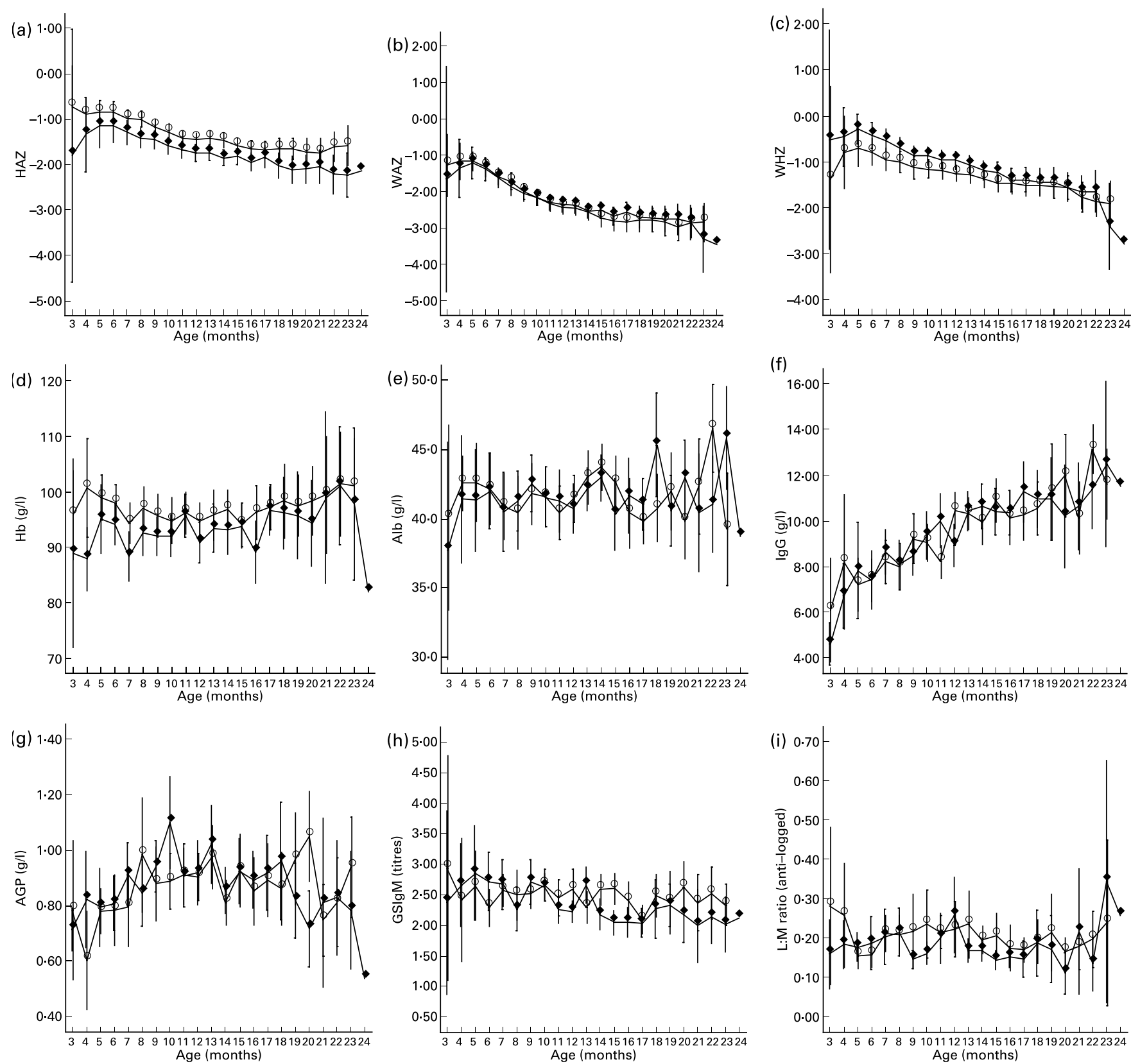

Fig. 1. Relationships of $z$-scores and biochemical variables with age in male $(\diamond)$ and female (O) infants. (a) Height-for-age $z$-score $(H A Z)$, (b) weight-for-age z-score (WAZ), (c) weight-for-height z-score (WHZ), (d) Hb, (e) albumin, (f) IgG, (g) $\alpha$-1-acid glycoprotein (AGP), (h) Giardia-specific IgM (GSIgM), (i) lactulose: mannitol (L:M) ratio. Values are means, with $95 \% \mathrm{Cl}$ represented by vertical bars.

antigens, such as toxins and organisms, and responded by a rise in $\mathrm{IgG}$ levels. In the Gambian infants, IgG levels were negatively associated with age-corrected growth rates $(r=-0.63 ; P<0.001)^{(5)}$. However, the present study did not show significant relationships between the $\mathrm{IgG}$ level and infants' growth, although all were negative (Table 2).

It is generally assumed that the concentration of AGP at birth is very low, but by 10 months of age ${ }^{(31)}$ infants are thought to have reached adult levels (range $0.5-1.0 \mathrm{~g} / \mathrm{l}^{(30)}$; mean 0.88 (SD 0.21$\left.) \mathrm{g} / \mathrm{l}^{(32)}\right)$. The present study also showed evidence of increasing AGP levels in the first year of life (Fig. 1). Several different assay techniques have been used and there is no agreed cut-off signifying an elevated level for AGP, for example, cut-offs of above $0.75 \mathrm{~g} / \mathrm{l}^{(33)}, 1.0 \mathrm{~g} / \mathrm{l}^{(34)}$ and $1.2 \mathrm{~g} / \mathrm{l}^{(35-37)}$. In the present study, $60 \%$ of infants had an AGP level above $0.75 \mathrm{~g} / \mathrm{l}$, $30 \%$ were above $1.0 \mathrm{~g} / \mathrm{l}$ and $16 \%$ were above $1.2 \mathrm{~g} / \mathrm{l}$ and there were increased levels of stunting and underweight in infants with higher concentrations of AGP. This finding is in accord with Filteau et al. ${ }^{(38)}$ who also reported that a higher plasma concentration of AGP was significantly negatively correlated with HAZ as well as WAZ in Ghanaian children below 5 years of age.

Intestinal permeability values in the Bangladeshi infants were higher than those found in a group of UK infants (geometric mean $0 \cdot 12)^{(39)}$, and nearly three-quarters of them 
Table 2. Relationships between $z$-scores and biochemical status after controlling for age and sex*

\begin{tabular}{|c|c|c|c|c|c|c|}
\hline \multirow{2}{*}{$\begin{array}{l}\text { Dependent variables... } \\
\text { Independent variables }\end{array}$} & \multicolumn{2}{|c|}{ HAZ } & \multicolumn{2}{|c|}{ WAZ } & \multicolumn{2}{|c|}{ WHZ } \\
\hline & B & $P$ & B & $P$ & B & $P$ \\
\hline $\mathrm{Hb}$ & 0.009 & $<0.001$ & 0.012 & $<0.001$ & 0.007 & 0.004 \\
\hline Albumin & 0.010 & 0.016 & 0.007 & NS & -0.002 & NS \\
\hline $\lg G$ & -0.006 & NS & -0.004 & NS & 0.004 & NS \\
\hline AGP & -0.194 & 0.011 & -0.263 & 0.005 & -0.116 & NS \\
\hline GSIgM & -0.040 & NS & -0.090 & 0.015 & -0.072 & 0.039 \\
\hline L:M ratio & -0.193 & 0.039 & -0.267 & 0.019 & -0.165 & NS \\
\hline
\end{tabular}

HAZ, height-for-age $z$-score; WAZ, weight-for-age $z$-score; WHZ, weight-for-height $z$-score; AGP, $\alpha$-1-acid glycoprotein; GSIgM, Giardia-specific IgM titre; L:M, lactulose:mannitol.

${ }^{*} \mathrm{~B}$ is the regression coefficient. WAZ showed a significant quadratic age effect, therefore quadratic age factor also controlled with linear age factor.

(62\%) showed evidence of intestinal mucosal damage compared with the UK level. The geometric mean of the $\mathrm{L}: \mathrm{M}$ ratio in the infants was $0 \cdot 15$ (Table 1), which is lower than the 0.23 found in a previous rural Bangladesh study ${ }^{(40)}$ although the children were older (2-5 years of age) and much lower than the Gambian mean of $0 \cdot 38^{(39)}$. Intestinal mucosal malfunction was also significantly associated with chronic malnutrition (HAZ and WAZ; $P=0.039$ and 0.019, respectively) rather than acute malnutrition. A previous study in Bangladesh ${ }^{(6)}$ with older children aged 2-5 years also found that better small-intestinal mucosal function was associated with improved WAZ after 8 and 12 months ( $r-0.19, P<0.02$ and $r-0.21, P<0.05$ for the changes at 8 to 12 months, respectively).

Giardiasis causes acute diarrhoea, so it is not surprising that Giardia infection was related to both acute weight loss in the present study (i.e. WAZ and WHZ; $P=0.015$ and 0.039 , respectively); Lunn et al. in The Gambia found that a shortterm weight reduction was associated with elevation of $\operatorname{GSIgM}^{(41)}$. However, the present study did not show a clear impact of high endemicity of Giardia infection on intestinal mucosal damage as measured by the L:M ratio.

In the present study, the prevalence of geohelminths in these infants ascertained by eggs in stools was consistently very low (2.8\% for Ascaris, $0.8 \%$ for Trichuris and no infection of hookworms) throughout the study. The egg counts of Ascaris and Trichuris infection were also very low according to the WHO classification ${ }^{(42)}$, even taking into account the posited lowered fecundity of female Ascaris worms in Bangladesh $^{(43)}$. It is therefore unlikely these very low levels of geohelminth infection would be important to the growth faltering observed in these infants.
The prevalence of Giardia using the GSIgM titre was consistently high, at $98 \%$, in the study. The very high endemicity suggested by the Giardia antibody titre contrasted greatly with the prevalence of Giardia cysts detected in stool examinations, which was very low during the study period. It is generally accepted that excretion of cysts is intermittent; thus a single stool sample, as used in the study, from an infant with a low-intensity infection might contain few, if any cysts, leading to under-diagnosis ${ }^{(44)}$. It is also important to appreciate that the two techniques are measuring different things: the GSIgM titre is a measure of the body's exposure to Giardia antigen whereas cysts are only produced when there is an active infection of Giardia trophozoites established in the intestine. These apparently contradictory results may be due to an interaction with breast-feeding, since anti-Giardial factors in the milk may inhibit trophozoite adherence to enterocytes, so reducing the likelihood of persistent infection ${ }^{(45)}$, whilst milk-borne leucocytes actively kill the organisms ${ }^{(46)}$. Consequently, breast-feeding children may be frequently exposed to this parasite but rarely develop a full episode of Giardiasis, so accounting for the differences between stool and serological estimates of Giardia prevalence.

In conclusion, infants in the present study showed a sharp decline in growth and nutritional status in the first 2 years of life and their immune system was frequently stimulated by infections such as Giardia. Infants also had damaged intestinal mucosal function and Giardia infection, in particular, and damaged mucosal function both associated with acute and chronic malnutrition. However, intestinal permeability levels were not directly associated with any of the inflammation markers or with Giardia infection. The elevation of Giardia-specific titres throughout the study is indicative of

Table 3. Relationships between biochemical variables after controlling for age and sex*

\begin{tabular}{|c|c|c|c|c|c|c|c|c|c|c|c|c|}
\hline \multirow{2}{*}{$\begin{array}{l}\text { Dependent variables... } \\
\text { Independent variables }\end{array}$} & \multicolumn{2}{|c|}{$\mathrm{Hb}$} & \multicolumn{2}{|c|}{ Albumin } & \multicolumn{2}{|c|}{$\lg G$} & \multicolumn{2}{|c|}{ AGP } & \multicolumn{2}{|c|}{ GSIgM } & \multicolumn{2}{|c|}{ L:M ratio } \\
\hline & B & $P$ & B & $P$ & B & $P$ & B & $P$ & B & $P$ & B & $P$ \\
\hline $\mathrm{Hb}$ & - & - & 0.013 & NS & -0.013 & NS & -0.003 & 0.002 & 0.001 & NS & 0.001 & NS \\
\hline Albumin & 0.056 & NS & - & - & 0.135 & $<0.001$ & 0.003 & 0.039 & 0.031 & $<0.001$ & -0.001 & NS \\
\hline $\lg G$ & -0.199 & NS & 0.569 & $<0.001$ & - & - & 0.034 & $<0.001$ & 0.080 & $<0.001$ & -0.002 & NS \\
\hline AGP & -3.190 & 0.002 & 1.070 & 0.036 & 2.568 & $<0.001$ & - & - & 0.416 & $<0.001$ & 0.028 & NS \\
\hline GSIgM & 0.042 & NS & 1.567 & $<0.001$ & 0.947 & $<0.001$ & 0.068 & $<0.001$ & - & - & -0.006 & NS \\
\hline L:M ratio & 1.385 & NS & -0.262 & NS & -0.212 & NS & 0.045 & NS & -0.056 & NS & - & - \\
\hline
\end{tabular}




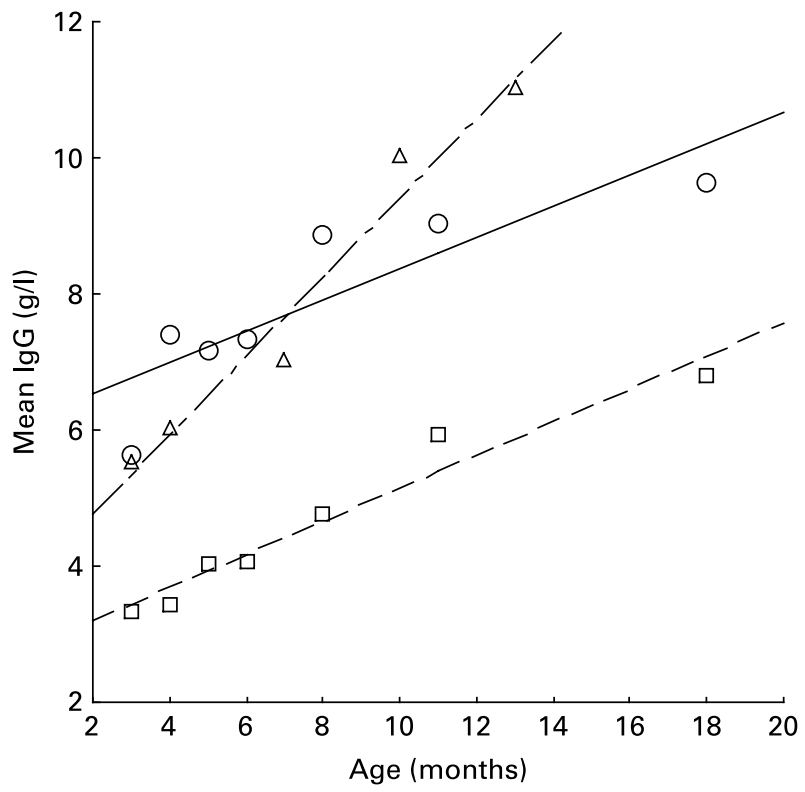

Fig. 2. The relationship between $\lg G$ and age in the UK $(---; \square)^{(30)}$, The Gambia (---; $\Delta)^{(5)}$ (LG Lunn, personal communication) and the present study $(-; \bigcirc)$

endemicity of Giardia infection. So, rural Bangladeshi infants are being exposed to high levels of infection with concomitant gut damage and growth faltering, presumably arising from inappropriate infant-feeding practices and poor standards of hygiene; a previous study in Bangladesh reported that less than $5 \%$ of infants below 6 months of age were exclusively breast-fed ${ }^{(47)}$. Bangladeshi mothers need to be much more aware of the importance of exclusive breastfeeding for the first 6 months as well as the necessity of better feeding and hygiene standards.

\section{Acknowledgements}

Financial support for the project was provided by the University of Cambridge, The Parkes Foundation and Fitzwilliam College, Cambridge. Logistical support was provided by Professor Kazuhiko Moji (Institute of Tropical Medicine, Nagasaki University, Japan), Professor Mahmudhur Rahman (National Institute of Preventive and Social Medicine (NIPSOM), Bangladesh) and Dr Rashidul Haque (International Centre for Diarrhoeal Disease Research, Bangladesh). We particularly thank our research staff, Dr Liton, Dolly, Ruma, Sherin, Sheuli, Mizan and Aneeka and all the mothers and infants who participated in the study. Helpful statistical support was provided by Dr Paul Callow (Computer Services, University of Cambridge) and Dr Yoichi Ito (Department of Biostatistics, University of Tokyo, Japan).

There are no conflicts of interest for any of the three authors. R. G. conceived of and designed the study, analysed and interpreted the data and drafted the paper. C. G. N. M.-T. conceived of and designed the study, analysed and interpreted the data and revised the paper. P. G. L. conceived of and designed the study, interpreted the data and revised the paper.

\section{References}

1. Helen Keller International \& Institute of Public Health and Nutrition (2006) Bangladesh in Facts and Figures: 2005 Annual Report of the Nutritional Surveillance Project. Dhaka, Bangladesh: Helen Keller International.

2. Shrimpton R, Victora CG, de Onis M, et al. (2001) Worldwide timing of growth faltering: implications for nutritional interventions. Pediatrics 107, e75.

3. The World Bank (2006) Repositioning Nutrition as Central to Development: A Strategy for Large-Scale Action. Washington, DC: The World Bank.

4. Lunn PG (2000) The impact of infection and nutrition on gut function and growth in childhood. Proc Nutr Soc 59, $147-154$.

5. Campbell DI, Elia M \& Lunn PG (2003) Growth faltering in rural Gambian infants is associated with impaired small intestinal barrier function, leading to endotoxemia and systemic inflammation. J Nutr 133, 1332-1338.

6. Northrop-Clewes CA, Rousham EK, Mascie-Taylor CGN, et al. (2001) Anthelmintic treatment of rural Bangladeshi children: effect on host physiology, growth, and biochemical status. Am J Clin Nutr 73, 53-60.

7. Goto R, Panter-Brick C, Northrop-Clewes CA, et al. (2002) Poor intestinal permeability in mildly stunted Nepali children: associations with weaning practices and Giardia lamblia infection. Br J Nutr 88, 141-149.

8. Raj SM, Sein KT, Anuar K, et al. (1996) Effect of intestinal helminthiasis on intestinal permeability of early primary schoolchildren. Trans $R$ Soc Trop Med Hyg 90, 666-669.

9. Tripathy K, Duque E, Bolanos O, et al. (1972) Malabsorption syndrome in ascariasis. Am J Clin Nutr 25, 1276-1287.

10. Gupta MC \& Urrutia JJ (1982) Effect of periodic antiascaris and antigiardia treatment on nutritional status of preschool children. Am J Clin Nutr 36, 79-86.

11. Crompton DWT \& Nesheim MC (2002) Nutritional impact of intestinal helminthiasis during the human life-cycle. Аnnu Rev Nutr 22, 35-59.

12. O'Lorcain P \& Holland C (2000) The public health importance of Ascaris lumbricoides. Parasitology 121, S51-S71.

13. Oberhelman RA, Guerrero ES, Fernandez ML, et al. (1998) Correlations between intestinal parasitosis, physical growth, and psychomotor development among infants and children from rural Nicaragua. Am J Trop Med Hyg 58, 470-475.

14. Goto R, Mascie-Taylor CGN \& Lunn PG (2008) Impact of antiGiardia and antihelmintic treatment on infant growth and intestinal permeability in rural Bangladesh: a randomised double-blind controlled study. Trans $R$ Soc Trop Med Hyg (epublication ahead of print version 11 September 2008).

15. Lohman TG, Roche AF \& Martorell R (1988) Anthropometric Standardization Reference Manual. Champaign, IL: Human Kinetic Books.

16. Ulijaszek SJ \& Kerr DA (1999) Anthropometric measurement error and assessment of nutritional status. Br J Nutr 82, $165-177$

17. Akram S, Mourani S, Ou C, et al. (1998) Assessment of intestinal permeability with a two-hour urine collection. Dig Dis Sci 43, 1946-1950.

18. World Health Organization (1994) Bench aids for the diagnosis of intestinal helminthes. Geneva: WHO.

19. Lunn PG, Northrop CA \& Northrop AJ (1989) Automated enzymatic assays for the determination of intestinal permeability probes in urine. 2. Mannitol. Clin Chim Acta 183, 163-170.

20. Northrop CA, Lunn PG \& Behrens RH (1990) Automated enzymatic assays for the determination of intestinal permeability probes in urine. 1. Lactulose and lactose. Clin Chim Acta 187, $79-88$. 
21. Goka AKJ, Rolston DDK, Mathan VI, et al. (1986) Diagnosis of Giardiasis by specific IgM antibody enzyme-linked immunosorbent assay. Lancet ii, 184-186.

22. Kuczmarski RJ, Ogden CL, Grummer-Strawn LM, et al. (2000) CDC Growth Charts: United States, Advance Data. Atlanta, GA: Centers for Disease Control and Prevention.

23. Panter-Brick C, Lunn PG, Goto R, et al. (2004) Immunostimulation and growth faltering in UK infants. Am J Hum Biol 16, 581-587.

24. Lydyard PM, Whelan A \& Fanger MW (2000) Immunology. Oxford: BIOS Scientific Publishers Ltd.

25. Laurell C-B (1985) Acute phase proteins - a group of protective proteins. In Recent Advances in Clinical Biochemistry, pp. 103-124 [CP Price and KGMM Alberti, editors]. Edinburgh: Churchill Livingstone.

26. Arifeen SE, Black RE, Caulfield LE, et al. (2000) Infant growth patterns in the slums of Dhaka in relation to birth weight, intrauterine growth retardation, and prematurity. Am J Clin Nutr 72, $528-535$

27. Karim E \& Mascie-Taylor CGN (2001) Longitudinal growth of Bangladeshi infants during the first year of life. Ann Hum Biol 28, 51-67.

28. Begum HA (2004) Effect of food supplementation on the growth of infants in rural Bangladesh. PhD thesis, University of Cambridge.

29. Janeway CA \& Travers P (1996) Immunobiology: the Immune System in Health and Disease, 2nd ed. Edingburgh: Current Biology Ltd/Garland Publishing Inc.

30. Meites S (1989) Pediatric Clinical Chemistry: Reference (Normal) Values. Washington, DC: AACC Press.

31. Calvin J, Neale G, Fotherby KJ, et al. (1988) The relative merits of acute phase proteins in the recognition of inflammatory condition. Ann Clin Biochem 25, 60-66.

32. Sann L, Bienvenu J, Lahet C, et al. (1981) Serum orosomucoid concentration in newborn infants. Eur J Pediatr 136, $181-185$

33. Filteau SM, Willumsen JF, Sullivan K, et al. (2000) Use of the retinol-binding protein:transthyretin ratio for assessment of vitamin A status during the acute-phase response. $\mathrm{Br} J$ Nutr 83, 513-520

34. Adelekan DA, Northrop-Clewes CA, Owa JA, et al. (2003) Use of biomarkers of sub-clinical infection, nutrition and neonatal maturity to interpret plasma retinol in Nigerian neonates. $\mathrm{Br} \mathrm{J}$ Nutr 90, 353-361.
35. Hautvast JLA, Tolboom JJM, Kafwembe EM, et al. (2000) Severe linear growth retardation in rural Zambian children: the influence of biological variables. Am J Clin Nutr 71, 550-559.

36. Paracha P, Jamil A, Northrop-Clewes CA, et al. (2000) Interpretation of vitamin A status in apparently healthy Pakistani children by using markers of subclinical infection. $A m J$ Clin Nutr 72, 1164-1169.

37. Wieringa FT, Dijkhuizen MA, West CE, et al. (2002) Estimation of the effect of the acute phase response on indicators of micronutrient status in Indonesian infants. $J$ Nutr 132, 3061-3066.

38. Filteau SM, Morris SS, Raynes JG, et al. (1995) Vitamin A supplementation, morbidity, and serum acute-phase proteins in young Ghanaian children. Am J Clin Nutr 65, 434-438.

39. Lunn PG, Northrop-Clewes CA \& Downes RM (1991) Intestinal permeability, mucosal injury, and growth faltering in Gambian infants. Lancet 338, 907-910.

40. Rousham EK, Northrop-Clewes CA \& Lunn PG (1998) Maternal reports of child illness and the biochemical status of the child: the use of morbidity interviews in rural Bangladesh. Br J Nutr 80, 451-456.

41. Lunn PG, Erinoso HO, Northrop-Clewes CA, et al. (1998) Giardia intestinalis is unlikely to be a major cause of the poor growth of rural Gambian infants. J Nutr 129, 872-877.

42. World Health Organization (2002) Prevention and Control of Schistosomiasis and Soil-Transmitted Helminthiasis. WHO Technical Report Series no. 912, Geneva: WHO.

43. Hall A \& Holland C (2000) Geographical variation in Ascaris lumbricoides fecundity and its implications for helminth control. Parasitol Today 16, 540-544.

44. Booth M, Vounatsou P, N'goran EK, et al. (2003) The influence of sampling effort and the performance of the Kato-Katz technique in diagnosing Schistosoma mansoni and hookworm co-infections in rural Côte d'Ivoire. Parasitology 127, 525-531.

45. Crouch AA, Seow WK, Whitman LM, et al. (1991) Effect of human milk and infant milk formulae on adherence of Giardia intestinalis. Trans R Soc Trop Med Hyg 85, 617-619.

46. Gillin FD (1987) Giardia lamblia: the role of conjugated and unconjugated bile salts in killing by human milk. Exp Parasitol 63, 74-83.

47. Arifeen S, Black RE, Antelman G, et al. (2001) Exclusive breastfeeding reduces acute respiratory infection and diarrhea deaths among infants in Dhaka slum. Pediatrics 108, E67. 